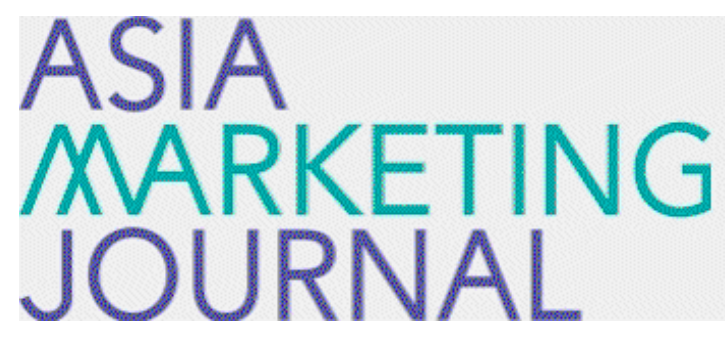

ASIA MARKETING JOURNAL

Volume 21 | Issue 1

Article 4

4-30-2019

\title{
The Effects of Variety and Visual Cue on Perceived Quantity and Consumer Attitude toward Participation into Sales Promotion Events
}

Changhyun Lee

Youngchan Kim

Follow this and additional works at: https://amj.kma.re.kr/journal

Part of the Marketing Commons

\section{Recommended Citation}

Lee, Changhyun and Kim, Youngchan (2019) "The Effects of Variety and Visual Cue on Perceived Quantity and Consumer Attitude toward Participation into Sales Promotion Events," Asia Marketing Journal: Vol. 21 : Iss. 1 , Article 4.

Available at: https://doi.org/10.15830/amj.2019.21.1.65

This Article is brought to you for free and open access by Asia Marketing Journal. It has been accepted for inclusion in Asia Marketing Journal by an authorized editor of Asia Marketing Journal. 


\title{
The Effects of Variety and Visual Cue on Perceived Quantity and Consumer Attitude toward Participation into Sales Promotion Events
}

\author{
Changhyun Lee* \\ Youngchan $\mathrm{Kim}^{* *}$
}

\begin{abstract}
Most studies on how people perceive a given quantity of items were conducted with visual cues exclusively and only offered spatial area based explanations, such as spatial estimation and perceptual grouping theories. This article establishes how people perceive a given quantity when only a written description is provided without any visual cues. Across two studies we show that variety decreases perceived quantity when a variety cue is given, while variety increases perceived quantity when a visual cue is not given. This is because people tend to rely heavily on spatial areas when a visual cue is present and because people are prone to confirmation bias when they are provided with no visual cues but only written descriptions. Furthermore, we highlight that quantity perception has a mediation effect on consumers' attitude- the intention to participate in sales promotional events. Lastly, we summarize the article and discuss its contributions, implications, limitations, and suggestions for future research.
\end{abstract}

Key words: Variety, perceived quantity, spatial area, spatial estimation, confirmation bias

\section{Introduction}

Imagine there are two grocery stores, store $\mathrm{A}$ and store B. If store A offers a bigger variety of products than store $B$, which store do you think would contain a larger quantity of products that it stocks? This time, imagine there are two restaurants $A$ and $B$. If restaurant $A$ serves a wider variety of items on the menu than restaurant $B$, which restaurant do you think can serve more meals? In real market settings, when consumers evaluate products, services, or brands, they like to use their time

\footnotetext{
* Graduate Student, Yonsei University

** Professor, Yonsei University (youngkim@yonsei.ac.kr), Corresponding Author
} 
more efficiently and therefore simplify evaluation tasks as much as they possibly can (Dehaene 1992, 1997; Tversky and Kahneman 1974; Yadav 1994; Piazza et al. 2004; Redden and Hoch 2009). However, this kind of predisposition of consumers may lead them to make inaccurate evaluations or biased decisions. For example, when we asked 40 participants the opening questions above in a pretest, the majority chose store $\mathrm{A}$ for the first question and restaurant $\mathrm{A}$ for the second question (85\% for store A, 90\% for restaurant $A$ ), even though they were not provided with enough information or had sufficient reason to make that decision.

In reality, the possibility of either store being the correct answer is fifty-fifty, which leads to the following question: why did the majority of people choose the variety answer option? From this biased decision-making behavior in quantity perception, we developed our research questions and possible explanations for these questions and conducted the two studies to support our proposed theoretical mechanism. In regard to quantity perception, there is an abundance of existing research. For example, previous studies demonstrated that people tend to perceive less quantity when variety is presented than otherwise and that they utilize spatial area to estimate quantity (Krueger 1972; Raghubir and Krishna 1996; Vos et al. 1988; Redden and Hoch 2009). Those studies are well-established in how people perceive quantity of the given picture cue condition.
Nonetheless, the literature remains limited since most of prior studies were conducted with visual cues exclusively and offered spatial area based explanations, such as spatial estimation and perceptual grouping theories. However, as more and more consumers prefer online shopping to offline shopping, consumers are now more likely to get sales promotional information or advertisement by written text messages, such as promotional e-mail messages, text messages, and even SNS messages. Therefore, how people perceive quantity of items when only written information is given without a visual cue is much more important matter to study. In this case, people are not able to use spatial area to examine quantity of products, so they must decide the quantity in some other ways. Many previous studies assume that more variety is often used as a cue for more quantity and that this notion is one of popular belief among people (Broniarczyk et al. 1998; Redden and Hoch 2009). In addition, when consumers evaluate products, services, or brands, they like to use their time more efficiently and thus simplify the evaluation tasks as much as they can (Dehaene 1992, 1997; Tversky and Kahneman 1974; Yadav 1994; Piazza et al. 2004; Redden and Hoch 2009). From these explanations, we posit that people would rely on their own belief to minimize their evaluation process when they decide the quantity of products. Furthermore, we assume that this mechanism would be more common when people are given only 
written description about quantity of products without a visual cue because people cannot use spatial area.

The primary objective of this article is to establish how people perceive given quantity when only the written description is presented without any visual cue. Additionally, we highlight that quantity perception has a mediation effect on consumers' attitude-intention to participate in sales promotional events. In this paper, we define the consumers' attitude as the degree to which consumers intend to participate in the sales promotional draw events. This research question has not been fully investigated since most previous research used quantity perception as a final dependent variable (Frith and Frith 1972; Ginsburg 1978; Vos et al. 1988; Frick 1987; Folkes and Matta 2004; Redden and Hoch 2009). We anticipate opposite results to Redden and Hoch's research (2009) when a visual cue is not given. That is, we posit that variety increases perceived quantity only when the written descriptive cue is given without a visual cue. We reason that if people cannot rely on spatial estimation because of the lack of a visual cue, they will simplify their evaluation tasks in cognitive ways, such as relying on previously held beliefs, expectations or desired conclusions, which is referred to as confirmation bias (Jonas et al. 2001). In two studies, this article verifies the role of perceived quantity as a mediator for consumers' attitude. The current study provides implications and directions for future research.

\section{Theoretical Background and Hypotheses}

\subsection{Spatial Estimation of Quantity and Variety}

A lot of previous research suggests that perceived quantity was studied mostly with a visual cue. For example, previous researchers gave some figures to estimate the number of items and measured the perceived quantity (Krueger 1972; Raghubir and Krishna 1996; Vos et al. 1988; Redden and Hoch 2009). In this experiment setting, the participants exclusively relied on spatial estimation since the visual cue was given (Redden and Hoch 2009). We use the term "spatial estimation" mainly as it connotes the behavior that people estimate the number of items with their bare eyes based on spatial area and the term "quantity" to indicate how much.

A myriad of studies with visual cues have suggested that people can quantify the items accurately and instantaneously up to six items (Kaufman et al. 1949) and that people often estimate the quantity (Dehaene 1992, 1997; Piazza et al. 2004) in a very short time (Mandler and Shebo 1982). Because of these predispositions, people are likely to perceive 
quantity inaccurately and incorrectly when there are more than six items. For example, people tend to perceive more dots when dots are displayed in enlarged area than in small area (Krueger 1972), and people are likely to perceive less quantity when variety exists (e.g., circle, triangle, star, square, and diamond shapes) than when variety does not exist (e.g., only circle shape) even though the actual quantity of the dots and figures for each condition (large area vs small area, variety vs no variety) is the same (Redden and Hoch 2009). These findings imply that the factors which affect perceived spatial area can affect perceived quantity as well, and that variety plays a role in reducing perceived spatial area by breaking up a whole (Redden and Hoch 2009). This is because people estimate the number of items based on the Gestalt figure (Firth and Frith 1972; Vos et al.1988; Redden and Hoch 2009). For example, people have difficulty in merging the items into a whole when different items are given, so they perceive the smaller spatial area of a whole and consequently perceive less quantity of items (Huang et al. 2006; Redden and Hoch 2009). However, this mechanism explains the effect of the presence of variety, only when a visual cue exists.

We anticipate that if people are unable to estimate based off of the spatial area with their bare eyes by the lack of a visual cue, they will simplify their evaluation tasks in their cognitive processes, such as relying on previously held beliefs, expectations or desired conclusions, which is referred to as confirmation bias (Jonas et al. 2001). In two studies, we show how people perceive the number of given items without a visual cue.

\subsection{Confirmation Bias and the Relationship between Variety and Quantity}

When consumers evaluate products, services, or brands, they like to use their time more efficiently and thus simplify the evaluation tasks as much as they can (Dehaene 1992, 1997; Tversky and Kahneman 1974; Yadav 1994; Piazza et al. 2004; Redden and Hoch 2009). Moreover, people prefer an alternative that requires less cognitive efforts to an alternative that requires more cognitive efforts (Garbarino and Edell 1997). Although consumers can reduce their efforts to search for information for evaluation by simplifying the evaluation tasks, they have a risk of making an inappropriate decision at the same time (Jonas et al. 2001). Simplifying often leads to several biases in decision making, and one of the biases that result from simplifying evaluation tasks is confirmation bias (Schul-Hardt 1997; SchulHardt et al. 2000; Yin et al. 2016).

Confirmation bias is a predisposition that people tend to over-weigh information that confirms their previously held beliefs, expectations, or desired conclusions (Snyder and White 1981; 
Nickerson 1998). In other words, this bias represents a tendency that people are likely to search for information that supports them to maintain their own position, even though the position is not verified thoroughly with all of the available information (Johnston 1996; Pinkley et al. 1995). However, this bias mechanism appears in numerous evaluation circumstances and therefore is likely to cause decision debacles (Janis 1982; Nemeth and Rogers 1996). For example, people favor information that confirms their beliefs about their social stereotypes (Nickerson 1998), expectations in negotiations (Pinkley et al. 1995), and their desired feedback about themselves (Frey 1981).

From these previous findings, we anticipate that the relationship between variety and quantity can be also applied to confirmation bias as many previous studies allude to this bias in relationship between quantity and variety (Broniarczyk et al. 1998; Redden and Hoch 2009). This is because there is often more quantity when variety is offered. To confirm that there is a commonly held belief among people in regard to the relationship between variety and quantity as we propose, we conducted a pretest. For the pretest, 40 participants were recruited using Amazon's Mechanical Turk. We asked the participants five questions that are related to the relationship between variety and quantity (e.g., "There are grocery stores, $A$ and B. If store A offers more variety of products than store $B$, which store do you think contains a larger quantity of products that it stocks?"). The pretest questions are shown in the Appendix. For all five questions, people were asked to choose between two options, store A or store B. Store A was a variety option, and store $\mathrm{B}$ was a no-variety option. The results indicate that, on the average, 83\% of the participants chose store A ( $n$ for store $\mathrm{A}=33, \mathrm{n}$ for store $\mathrm{B}=7$ ).

This result indicates that there is a common belief or expectation of the relationship between variety and quantity, so we anticipate that it is by confirmation bias that people tend to perceive more quantity when variety is presented than otherwise, which seems to be the opposite to what previous research claimed (Redden and Hoch 2009). However, we assume that this predicted outcome is feasible only when there is no visual cue, on which researchers have mostly focused in their studies on perceived quantity. This is because people rely exclusively on spatial estimation with their bare eyes when a visual cue is given (Redden and Hoch 2009), rather than cognitive simplification.

\subsection{Mediation Effect of Perceived Quantity and Consumers' Attitude}

Perceived quantity has been used as a final dependent variable in the literature (Frith and Frith 1972; Ginsburg 1978; Vos et al. 1988; Frick 1987; Folkes and Matta 2004; Redden and Hoch 2009), and there is lack of research 
addressing what perceived quantity affects using perceived quantity as a mediator for consumers' attitude. In this article, we suggest that perceived quantity can play a role as a mediator for consumers' attitude since in the marketing industry there are many possible occasions where more perceived quantity can result in positive consumer attitudes. For example, if people perceive more quantity of reward products of a draw during a sales promotional event, they will be more likely to have higher participation intention in the event. In addition, if a specific packaging method leads people to perceive more quantity of items even though only the packaging style is different among products, sellers will definitely increase consumers' purchase intention by using this method. Therefore, to verify this mediation effect on consumers' attitude, we use a sales promotional event in the form of a draw event in two studies.

The first study shows that the presence of variety increases perceived quantity of items when a visual cue is not presented and that this increased perceived quantity brings greater participation intention in the draw event. In the second study, we tried to rule out possible alternative explanations from the first study. For example, participation intention of an event might be affected by the variety-seeking tendencies rather than perceived quantity. Alternatively, it could be that perceived quantity is affected by variety-seeking tendencies. Surplus to this, people could be able to quantify items correctly with a regularly arranged set of items, so they may actually count rather than estimate. To rule out these potential explanations, we measure variety-seeking behavior with two widely used scales measuring variety-seeking tendencies (Baumgartner and Steenkamp 1996; Donthu and Gilliland 1996; Jeong et al. 2016) and use an irregularly arranged set of items. Figure 1 represents our overall research framework, and we come up with following hypotheses:

\section{$H_{1}$ : When a visual cue is presented, variety will reduce the perceived quantity of items.}

〈Figure 1〉 Overall Research Framework

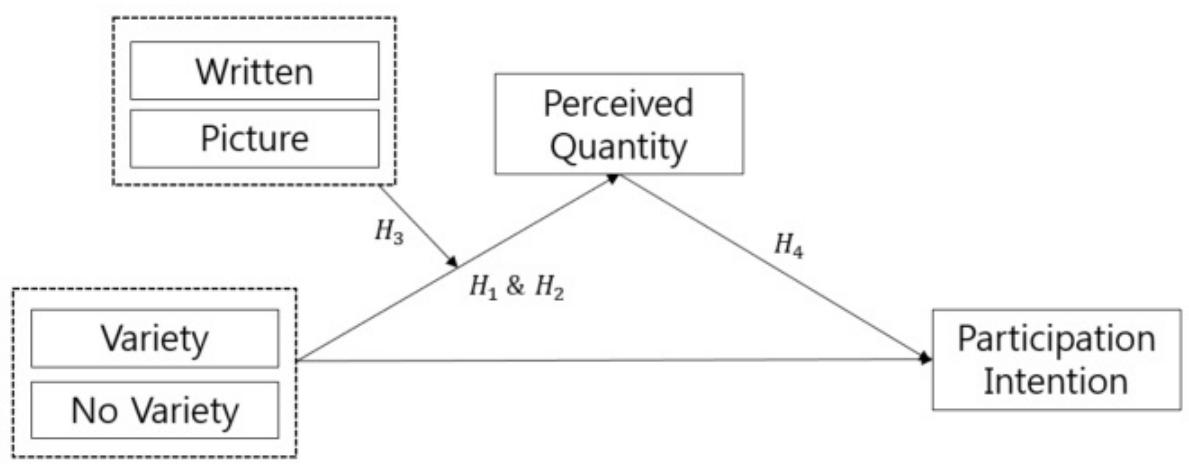

70 ASIA MARKETING JOURNAL Vol.21 No.01 April 2019 
$H_{2}$ : When a written description is presented without a visual cue, variety will increase the perceived quantity of items.

$H_{3}$ : There will be an interaction between the presence of variety and the presence of visual cue on perceived quantity.

$H_{4}$ : Perceived quantity that is resulted from the interaction between the presence of variety and the presence of visual cue plays a role as a mediator for consumers' attitude; in other words, the more quantity people perceive, the greater the participation intention in sales promotional draw events.

\section{Study 1}

The first study provides an exploration of whether the presence of variety influences participation intention in a sales promotional draw event. Variety should increase (decrease) the perceived quantity of items when a visual cue does not exist (does exist), and this increased (decreased) perceived quantity makes people more (less) willing to participate in the event.

For the stimulus we used paper notebooks, and we differentiated the variety and the novariety condition by color: the variety condition is comprised of twenty notebooks with different colors (navy, red, green, grey, and purple; four of each color) and the no-variety condition contains twenty notebooks of the same color (navy). All other things, asides from color, were identical. The colors that we choose for study 1 and subsequent studies were selected from a pretest that measured a degree of preference of each color to rule out the effect of color preference. Consequently, we got navy, red, green, grey, and purple for our studies. These five colors have no difference each other in the color preference measures $\left(M_{\text {navy }}\right.$ $=4.75, M_{\text {red }}=3.99, M_{\text {green }}=4.34, M_{\text {grey }}=$ 4.36, $\left.M_{\text {purple }}=4.17 ; p>0.3\right)$.

\subsection{Method}

One hundred seventy-one participants from Amazon's Mechanical Turk (MTurk) completed an online survey in exchange for a monetary reward $\left(M_{a g e}=41,63 \%\right.$ Female $)$. The participants were randomly assigned to each condition in a 2 (the presence of variety: variety vs no-variety) $\times 2$ (the presence of a visual cue: written vs picture) between-subjects design. First, participants were given with different stimulus for each condition. In the variety condition participants were provided with a stimulus that contains twenty notebooks with five different colors (navy, red, green, grey, and purple; four of each color), and in the no-variety condition participants were provided with twenty navy notebooks. In addition, participants assigned to the written 
condition got information on the presence of variety (variety or no-variety) from a written description without a visual cue, and participants who were assigned to the picture condition were given information via a brief written description with a visual cue. Specifically, in the picture condition, we did not provide the participants any direct information about variety and quantity of notebooks in order to let them count or estimate the quantity of the items from the given visual cue. Then all participants were presented with the same scenario, a sales promotional draw event in a stationery shop on a weekday. For the used stimuli, please refer to the appendix. Second, to test our theoretical mechanism, participants rated the degree of the perceived quantity of the notebooks being given out as a prize (Do you feel that the number of the prize notebooks being given out is $\cdots ; 1=$ Very little, $7=\mathrm{A}$ lot), and we measured the perceived level of variety as a manipulation check (How much prize variety do you think is in the event?; 1 = No variety, $7=\mathrm{A}$ lot of variety). Finally, participants completed our dependent variable, willingness to participate in the event (Are you willing to purchase more than $\$ 10$ to participate in the event?; 1 = Not at all, 7 = Very willing). Additionally, to rule out possible alternative explanations, participants completed a number of covariate questions using a seven-point Likert scale (Do you often participate in draw event? (OFTEN); Do you like drawing events?
(LIKE); If you participate in draws, do you tend to be drawn out often? (LUCK); How many people do you think usually come to the stationery shop on a weekday? (COME)).

\subsection{Results}

\subsubsection{Manipulation Checks}

As we expected, participants reported a higher degree of the presence of variety in the variety condition than in the no-variety condition ( $M_{\text {total-variety }}=3.60, M_{\text {total-variety }}=$ 1.63; $F(1,169)=66.23, p<0.01)$. Therefore, the manipulation of the presence of variety was successful.

\subsubsection{Perceived Quantity}

There was no difference between the conditions in the possible covariate measures other than LUCK as they were randomly assigned $\left(F_{\text {OFTEN }}(3,167)=1.12, p>.33\right.$; $F_{\text {LIKE }}(3,167)=.90, p>.44 ; F_{C O M E}(3,167)=$ $1.01, p>.95)$. We conducted an ANCOVA with the presence of variety as the independent variable, the presence of a visual cue as moderator, and perceived quantity as the dependent variable controlling for LUCK. There was no main effect of the presence of variety $(F(1,166)=1.58, p>.21)$. The analysis revealed an interaction between the presence of variety and the presence of a visual cue 
$(F(1,166)=7.64, p<.01)$. This interaction indicated that variety increased perceived quantity within the written condition $\left(M_{\text {written-variety }}=\right.$ 4.76, $M_{\text {written-no variety }}=3.75 ; \mathrm{F}(1,166)=7.61$, $\mathrm{p}<.01)$ and that variety did not significantly reduce perceived quantity within the picture condition ( $M_{\text {picture-variety }}=3.86, M_{\text {picture-no variety }}$ $=4.16 ; \mathrm{F}(1,166)=1.20, \mathrm{p}>.27)$. It seems that $H_{2}$ is supported whereas $H_{1}$ is not. Additionally, the written condition shows higher perceived quantity than the picture condition within the variety condition $\left(M_{\text {picture-variety }}=\right.$ 3.86, $M_{\text {written-variety }}=4.76 ; \mathrm{F}(1,166)=7.74, \mathrm{p}<$ .01 ), but there was no visible difference within the no-variety condition ( $M_{\text {picture-no variety }}=4.16$, $\left.M_{\text {Written-no variety }}=3.75 ; \mathrm{F}(1,166)=1.29, \mathrm{p}\right\rangle$ .25). According to the data the written-variety condition showed the highest perceived quantity among the conditions. The results are summarized in figure 2.

\subsubsection{Participation Intention}

We also conducted an ANCOVA with the presence of variety as the independent variable, the presence of a visual cue as moderator, and participation intention as the dependent variable controlling for LUCK and it revealed a similar pattern to above. There was no main effect of the presence of variety $(F(1,166)=.72, p>$ .39). The analysis revealed interaction between the presence of variety and the presence of a visual cue $(F(1,166)=4.61, p<.05)$. This interaction indicated that variety increased participation intention within the written condition $\left(M_{\text {written-variety }}=4.31, M_{\text {written-no variety }}=3.43\right.$; $\mathrm{F}(1,166)=4.23, \mathrm{p}<.05)$ and that variety did not significantly reduce participation intention

〈Figure 2〉 Effects of Variety on Perceived Quantity

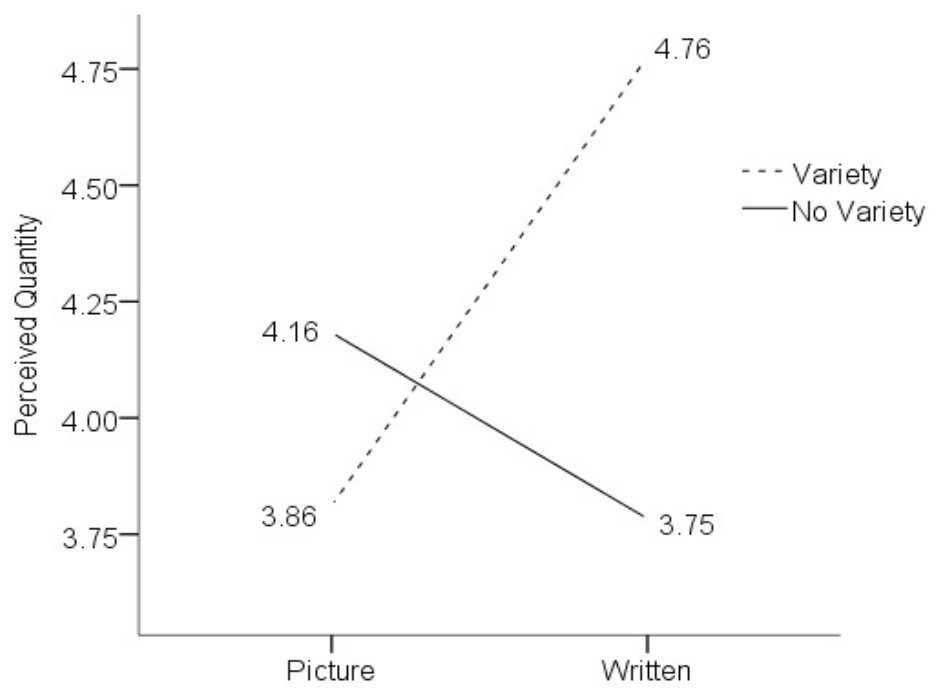

The Effects of Variety and Visual Cue on Perceived Quantity and Consumer Attitude toward Participation into Sales Promotion Events 73 
〈Figure 3〉 Effects of Variety on Participation Intention

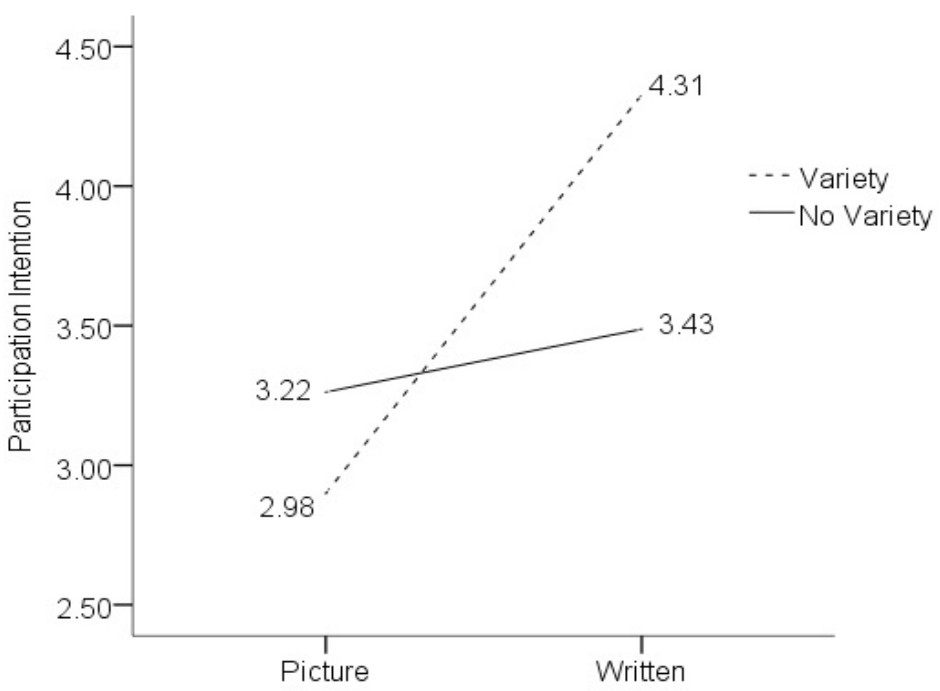

within the picture condition $\left(M_{\text {picture-variety }}=\right.$ 2.98, $M_{\text {picture-no variety }}=3.22 ; \mathrm{F}(1,166)=.89$, p >.34). Additionally, the written condition had higher participation intention than the picture condition within the variety condition $\left(M_{\text {picture-no variety }}=2.98, M_{\text {Written-variety }}=4.31\right.$; $\mathrm{F}(1,166)=13.18, \mathrm{p}<.001)$, but there was no difference between the two in the no-variety condition ( $M_{\text {picture-no variety }}=3.22, M_{\text {written-no variety }}$ $=3.43 ; \mathrm{F}(1,166)=.32, \mathrm{p}>.57)$. This similar pattern implies that there exists a mediation effect of perceived quantity on participation intention. The results are summarized in figure 3.

\subsubsection{Mediation}

As we expected, results from a moderated mediation analysis (with the presence of variety as the independent variable, the presence of a visual cue as moderator, the perceived quantity as mediator, and participation intention as the dependent variable controlling for LUCK; Hayes 2013, model 7: 5000 bootstrapped samples) demonstrate that perceived quantity acted as mediator of variety's effects on participation intention. The presence of a visual cue moderated the effects of variety on perceived quantity $(\beta=1.36$, SE $=.49, p<.01)$, and more perceived quantity resulted in increased participation intention $(\beta=.46, \mathrm{SE}=.08$, $p<.001)$. Within the written condition, variety increased perceived quantity, and thus, with this increased perceived quantity also rose participation intention (Conditional indirect effect $=.46$, Boot $\mathrm{SE}=.18 ; 95 \%$ confidence intervals (CIs), .13 to .87). However, within the picture condition, there was no visible effects on participation intention because of 
the lack of a significant difference between the variety and the no-variety condition in perceived quantity (Conditional indirect effect $=-.17$, Boot $\mathrm{SE}=.15 ; 95 \%$ CIs, -.50 to .10$)$. The analysis indicated that moderated mediation was successful (95\% CIs, .19 to 1.18).

\subsection{Discussion}

Consistent with our theories, study 1 reveals that there is moderated mediation. The writtenvariety condition increased the perceived quantity and thus rose participation intention. However, unlike our predictions and previous research (Redden and Hoch 2009), the picture-variety condition did not reduce perceived quantity significantly. Thus, there was an insignificant difference in perceived quantity between the picture-variety and the picture-no-variety condition as well as in participation intention.

We speculate two possible reasons for this. First, the participants who were assigned to the picture condition might be able to count the exact number of items with an ease since we gave a regularly arranged set of items. So, if they were more likely to quantify rather than estimate the quantity, our proposed mechanism would be unlikely to happen. Second, the participants might not completely rely on the spatial area to estimate the quantity of items because they had a clue about the total quantity in the brief written description. However, the latter is inevitable in our theoretical model as we have to make the conditions identical except for the key variable, the presence of variety. For example, it is impossible to create a written condition that describes all of the information about the given items without giving away any clue of the total quantity of the item.

Consequently, in study 2, we used a picture of a randomly arranged set of items to enhance our theoretical mechanism. Also, we measured variety-seeking tendencies to rule out the possible alternative explanation in study 2 since people might perceive more quantity and have higher participation intention because of their tendency of variety-seeking.

\section{Study 2}

As we state in the discussion section of study 1 , we used a picture of randomly arranged notebooks for the stimulus to strengthen our theoretical mechanism in study 2. In addition, we tried to rule out the effect of varietyseeking tendencies by adding a measure of it in the covariate analysis.

\subsection{Method}

One hundred eighty-one participants were recruited using Amazon's Mechanical Turk (MTurk) to complete an online survey in exchange for money ( $M_{\text {age }}=39,57 \%$ Female $)$. 
The participants were then randomly assigned to the same two-by-two between-subject design as study 1. Participants who were assigned to picture conditions were given with a picture of randomly arranged notebooks rather than a picture of regularly arranged notebooks used in study 1. All the other given stimuli in all conditions were identical to study 1 . Additionally, the procedure of this study was also identical to study 1, except for the measurement of variety-seeking tendencies at the end of the study. We measured variety-seeking behavior with two widely used scales measuring varietyseeking tendencies (Baumgartner and Steenkamp 1996; Donthu and Gilliland 1996; Jeong et al. 2016). The participants completed a nine-item measure of variety-seeking tendencies. The stimuli and measurement scales are provided in the Appendix.

\subsection{Results}

\subsubsection{Manipulation Checks}

Like study 1, study 2 also satisfied manipulation checks. The participants reported a higher degree of the presence of variety in the variety condition than in the no-variety condition $\left(M_{\text {total-variety }}=3.08, M_{\text {total-no variety }}=2.32\right.$; $F(1,179)=8.46, p<0.01)$. Therefore, the manipulation of the presence of variety was successful.

\subsubsection{Perceived Quantity}

We used not only LUCK as a covariate in the analysis but also variety-seeking tendencies. We averaged the scores of the nine items measuring variety-seeking tendencies to use in the analysis. Similar to study 1, there was no significant difference in the other possible covariate measures between the conditions as the random assignment was successful $\left(F_{\text {OFTEN }}(3,177)=1.25, p>.29 ; F_{\text {LIKE }}(3,177)\right.$ $\left.=1.55, p>.20 ; F_{\text {COME }}(3,177)=.80, p>.49\right)$. We conducted an ANCOVA with the presence of variety as the independent variable, the presence of a visual cue as moderator, and perceived quantity as the dependent variable controlling for LUCK and variety-seeking tendencies. The main effect of the presence of variety did not exist $(F(1,175)=.44, p>$ .50). The analysis indicated that there was an interaction between the presences of variety and the presence of a visual cue $(F(1,175)=$ 9.80, $p<.01)$. While variety increased perceived quantity within the written condition $\left(M_{\text {written-variety }}\right.$ $=4.67, M_{\text {written-no variety }}=3.86 ; \mathrm{F}(1,175)=$ $7.51, \mathrm{p}<.01)$, variety marginally reduced perceived quantity within the picture condition $\left(M_{\text {picture-variety }}=3.52, M_{\text {picture-no variety }}=4.05\right.$; $\mathrm{F}(1,175)=2.93, \mathrm{p}<.10)$. The results revealed that $H_{2}$ was supported significantly, while $H_{1}$ was supported marginally. In addition, the written condition showed higher perceived quantity than the picture condition within 
the variety condition $\left(M_{\text {picture-variety }}=3.52\right.$, $M_{\text {written-variety }}=4.67 ; \mathrm{F}(1,175)=13.69, \mathrm{p}<$ $.001)$, but there was no difference between them within the no-variety condition ( $M_{\text {picture-no variety }}$ $=4.05, M_{\text {written-no variety }}=3.86 ; \mathrm{F}(1,175)=$ $.56, \mathrm{p}>.45)$.

\subsubsection{Participation Intention}

Participation intention had a similar pattern of results. We also conducted an ANCOVA with the presence of variety as the independent variable, the presence of a visual cue as moderator, and participation intention as the dependent variable controlling for LUCK and variety-seeking tendencies. The main effect of the presence of variety was not qualified ( $F(1$, $175)=2.14, p>.14)$, but the interaction between the presence of variety and the presence of a visual cue was significant ( $F(1$, $166)=4.61, p<.05)$. This interaction revealed that variety increased participation intention within the written condition $\left(M_{\text {written-variety }}=\right.$ 4.07, $M_{\text {written-no variety }}=3.15 ; \mathrm{F}(1,175)=7.21$, $\mathrm{p}<.01$ ) and that variety did not significantly reduce participation intention within the picture condition $\left(M_{\text {picture-variety }}=2.85, M_{\text {picture-no variety }}=\right.$ 3.03: $\mathrm{F}(1,175)=.30, \mathrm{p}>$.58). Additionally, the written condition's participation intention was higher than the picture condition's variety condition $\left(M_{\text {picture-variety }}=2.85, M_{\text {written-variety }}=\right.$ 4.07; $\mathrm{F}(1,175)=10.74, \mathrm{p}<.01)$, but participation intention was about the same between the two conditions in the no-variety condition $\left(M_{\text {picture-no variety }}=3.03, M_{\text {written-no variety }}=3.15\right.$; $\mathrm{F}(1,175)=.003, \mathrm{p}>.95)$. The results are summarized in figure 5 .

〈Figure 4〉 Effects of Variety on Perceived Quantity

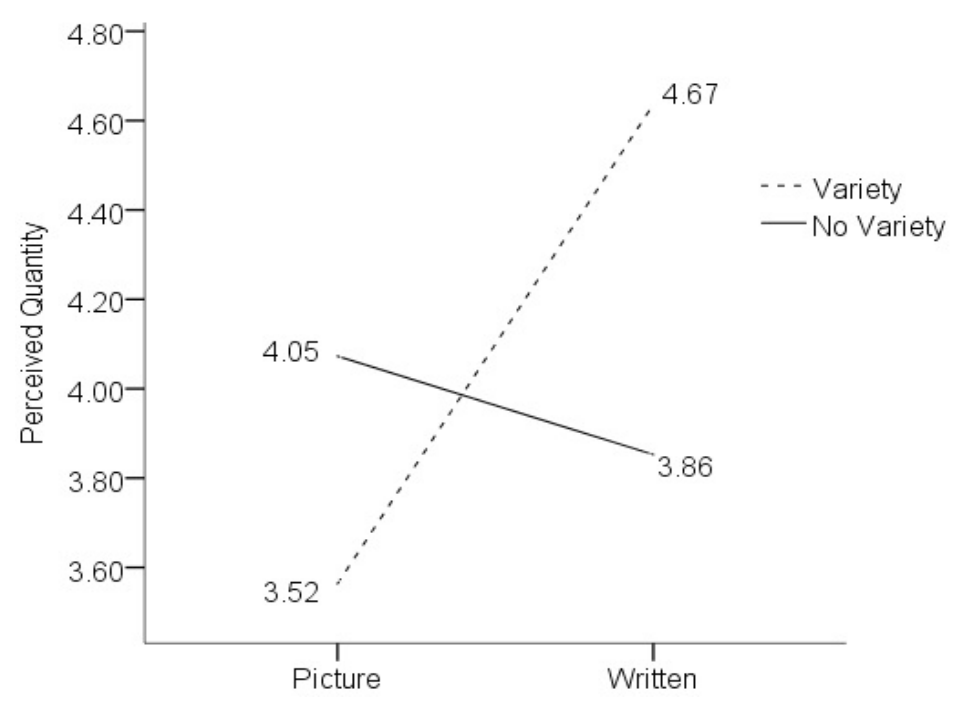

The Effects of Variety and Visual Cue on Perceived Quantity and Consumer Attitude toward Participation into Sales Promotion Events 77 
〈Figure 5〉 Effects of Variety on Participation Intention

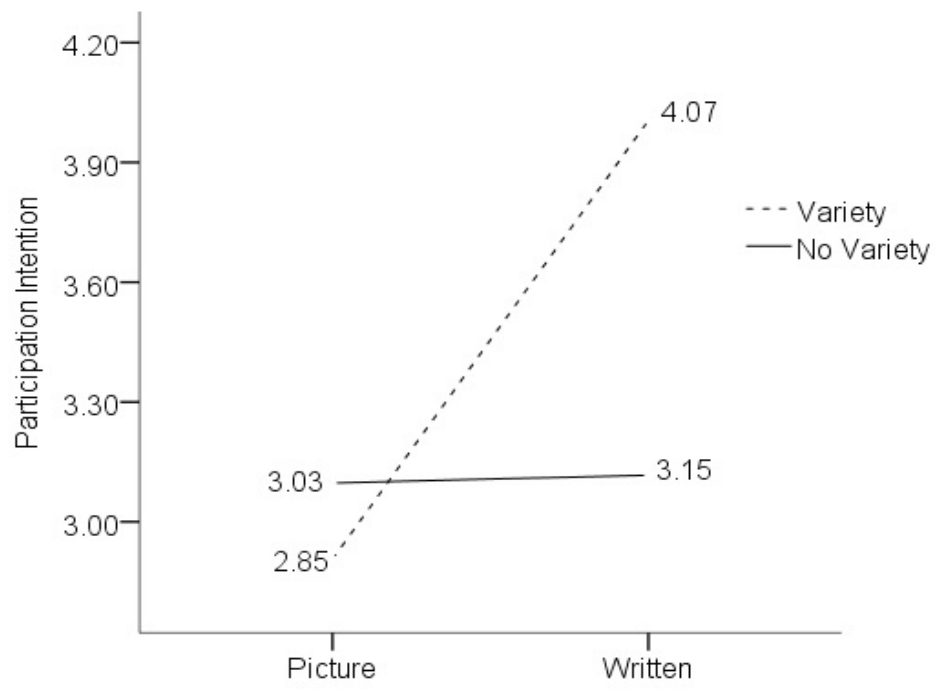

\subsubsection{Mediation}

As we predicted, results from the moderated mediation analysis (with the presence of variety as the independent variable, the presence of a visual cue as moderator, the perceived quantity as mediator, and participation intention as the dependent variable controlling for LUCK and variety-seeking tendency; Hayes 2013, model 7: 5000 bootstrapped samples) showed that perceived quantity played a role as a mediator in our proposed theoretical model. The effects of variety on perceived quantity were moderated by the presence of a visual cue $(\beta=1.29$, SE $=.41, \mathrm{t}=3.13, p<.01)$, and more perceived quantity resulted in increased participation intention $(\beta=.25, \mathrm{SE}=.08, \mathrm{t}=2.99, p<.01)$. Within the written condition, variety increased perceived quantity, and with this increased perceived quantity also rose participation intention (Conditional indirect effect $=.19$, Boot $\mathrm{SE}=.10 ; 95 \%$ confidence intervals (CIs), .03 to .47). However, within the picture condition, there was no effects on participation intention because the difference in perceived quantity between the variety and the no-variety condition was too marginal (Conditional indirect effect $=-.12$, Boot $\mathrm{SE}=.09 ; 95 \%$ CIs, -.37 to .002). The analysis indicated that the moderated mediation was qualified (95\% CIs, .07 to .72 ).

\subsection{Discussion}

The results of study 2 were consistent with the results of study 1 even though we controlled for the effects of variety-seeking tendencies. Specifically, variety increased perceived quantity 
within the written conditions and moderated mediation was still significant. Thus, more perceived quantity resulted in increased participation intention. However, in regard to the picture conditions, variety only marginally reduced perceived quantity, and it consequently made no difference in the participation intention between the variety and the no-variety condition. Despite these outcomes, perceived quantity and participation intention within the picture conditions remained less when variety was presented, which implies that less perceived quantity and participation intention occurred when variety was given. Therefore, the results of study 2 demonstrated that not only $\mathrm{H}_{2}, \mathrm{H}_{3}$, and $H_{4}$ were supported significantly, but $H_{1}$ was also supported marginally.

\section{General Discussion}

\subsection{Implications and Contributions}

Perceived quantity has been used only as a final dependent variable and studied only with a visual cue in the literature. For example, Redden and Hoch (2009) examined the relationship between variety and perceived quantity using diverse visual cues. They proposed that variety leads people to perceive less quantity since people heavily rely on the spatial area to make quantity judgments (Krueger 1972; Raghubir and Krishna 1996; Vos et al. 1988; Redden and Hoch 2009). Additionally, previous studies examined their mechanisms with simple random patterns of colored dots or common shapes without any marketing setting or scenario.

To extend and complement previous studies, this article studied how variety affected perceived quantity when only written descriptions of the stimuli are provided without a visual cue, where we predicted that there would be an interaction between the written and the visual condition because of the opposite directions of the outcomes. Furthermore, this article was conducted in market settings, such as sales promotional draw events, and thus we developed the studies to show that perceived quantity acts as a mediator for consumers' attitude and participation intention in events. Therefore, through two studies we indicated that variety without a visual cue increases perceived quantity and that this increased perceived quantity consequently raised people's participation intention in the events. Additionally, we introduced confirmation bias as the theoretical explanation for the effects of variety within the written conditions. People perceive more quantity of items because they have a common belief or expectation of the relationship between variety and quantity; people believe or expect that there will be more quantity when more variety exists. The pretest and following studies supported this proposed theoretical explanation. Therefore, academically, our findings extend the research 
scope by experimenting with and without a visual cue at the same time in marketing settings. Moreover, this article offers suggestions for real sales market industries as well. Based off the results of previous studies, firms might think that variety reduces perceived quantity in everyday circumstances, and thus apply the same mechanism even to situations where information about the number of items is conveyed only via a written description without a visual cue. However, this article proposes that variety has opposite effects on perceived quantity in such circumstances, so firms should operate strategic methods using a variety depending on whether a visual cue is given or not. For example, if stores try to promote their sales by holding sales promotional draw events with a photo of the reward products or with the presentation of the actual reward, they can attract more consumers and achieve their goal successively by making reward products have less variety. However, if stores hold the events giving information about reward only through a written description, the reward products must be varied to encourage a higher participation volume. For advertisement, if an insurance company sends promotional e-mail messages, text messages, or SNS messages about benefits of a new insurance product to their customers without a visual cue, it will make the customers think that the new product offers many benefits and finally lead them to purchase the product. Additionally, when electronic companies advertise brand new devices, giving written information on the functions of the devices can make customers feel that the devices offer them more functions and consequently bring better outcomes in consumers' attitude and sales. In fact, this article's suggestions are essentially based on products which have no differences from each other.

Therefore, we predict that the proposed effects of variety have a stronger impact on everyday products, which are likely to have little differences among them. The findings of this study can be also used in various sales promotional circumstances, such as online shopping events or coupon events, since written description without a visual cue is still used prominently in many cases. In sum, this article provides not only theoretical implications to academic fields but also practical implications to sales market industries.

\subsection{Limitations and Future Research}

In this article, we used a draw situation for our studies, so it was unavoidable not giving any clues of the total quantity of items as we had to make all of the conditions identical other than our key variable, namely the presence of variety. Thus, we were not able to fully support the previous studies' theoretical mechanism within the picture conditions. Future researchers need to make narrower context setting to develop a more sophisticated research. 
Additionally, this article did not show at what level of quantity the effect of variety is attenuated or disappears. In fact, because people can quantify the items accurately and instantaneously up to six items (Kaufman et al. 1949), they will not rely completely on spatial estimation or confirmation bias at different levels of quantity. For example, if people are given with only five items, which is a very small number, there could be no difference in perceived quantity between the variety and the no-variety condition since people can easily quantify. Therefore, to make more elaborate understanding of proposed mechanism more elaborate, it is necessary to find a point at which the effect of variety might be not significant.

Finally, this article used pictures of notebooks as visual cues. However, the results could be better supported or different if real products are given to participants for judging quantity. People might not be able to access given products' dimension, such as height and width, by seeing pictures of the products. If so, the effects of variety in the context of visual cues could be weakened. For instance, if people see actual notebooks rather than pictures of notebooks, the mechanism of spatial estimation could be more significant since they are able to judge quantity with more accurate dimensions of the notebooks. As a result, it would be beneficial to use actual products in future research to make the proposed theoretical mechanism more robust.

〈Received January 11. 2019〉

〈Accepted April 5. 2019〉

\section{References}

Baumgartner, H. and J. E. M. Steenkamp (1996), "Exploratory Consumer Buying Behavior: Conceptualization and Measurement," International Journal of Research in Marketing, 13, 121-137.

Broniarczyk, Susan M., Wayne D. Hoyer, and Leigh McAlister (1998), “Consumers' Perceptions of the Assortment Offered in a Grocery Category: The Impact of Item Reduction," Journal of Marketing Research, 35 (2), 166-176.

Dehaene, Stanislas (1992), "Varieties of Numerical Abilities," Cognition, 44, 1-42. (1997), The Number Sense: How the Mind Creates Mathematics, New York: Oxford University Press.Donthu, N. and D. Gilliland (1996), "The Infomercial Shopper," Journal of Advertising Research, 36, 69-76.

Donthu, N. and D. Gilliland (1996), "The Infomercial Shopper," Journal of Advertising Research, 36, 69-76.

Folkes, Valerie and Shashi Matta (2004), "The Effect of Package Shape on Consumers' Judgments of Product Volume: Attention 
as a Mental Contaminant," Journal of Consumer Research, 31 (2), 390-401.

Frey, D. (1981), “The Effect of Negative Feedback About Oneself and Cost of Information on Preferences for Information About the Source of This Feedback," Journal of Experimental Social Psychology, 17, 42-50.

Frick, Robert W. (1987), “The Homogeneity Effect in Counting," Perception and Psychophysics, 41 (1), 8-16.

Frith, Christopher D. and Uta Frith (1972), "The Solitaire Illusion: An Illusion of Numerosity," Perception and Psychophysics, 11 (6), 409-410.

Garbarino, Ellen C. and Julie A. Edell (1997), "Cognitive Effort, Affect, and Choice," Journal of Consumer Research, 24 (9), 147-158.

Ginsburg, Norman (1978), "Perceived Numerosity, Item Arrangement, and Expectancy," American Journal of Psychology, 91 (2), 267-273.

Huang, Liqiang, Hal Pashler, and Anne Treisman (2006), "Can We Select Two Colors Simultaneously?" Journal of Vision, 6 (6), 1101a.

Janis, I. L. (1982), "Groupthink (2nd ed., Rev.)," Boston: Houghton Mifflin, 235-246.

Jeong, H. G., Kate Christensen, and Aimee Drolet (2016), The Short-Lived Benefits of Variety Seeking Among the Chronically Indecisive," Journal of Experimental
Psychology, 22 (4), 423-435.

Johnston, L. (1996), "Resisting Change: Informationseeking and Stereotype Change," European Journal of Social Psychology, 26, 799-825. Jonas, Eva, Stefan Schulz-Hardt, Dieter Frey, and Norman Thelen (2001), "Confirmation Bias in Sequential Information Search After Preliminary Decisions: An Expansion of Dissonance Theoretical Research on Selective Exposure to Information," Norman Journal of Personality and Social Psychology, 80 (4), 557-571.

Kaufman, E. L., M. W. Lord, T. W. Reese, and J. Volkman (1949), "The Discrimination of Visual Number," American Journal of Psychology, 62, 498-525.

Krueger, Lester E. (1972), "Perceived Numerosity," Perception and Psychophysics, 11, 5-9. Mandler, George and Billie Jo Shebo (1982), "Subitizing: An Analysis of Its Component Processes," Journal of Experimental Psychology: General, 111 (1), 1-22.

Nemeth, C. J., and J. Rogers (1996), Dissent and the Search for Information," British Journal of Social Psychology, 35, 67-76.

Nickerson, Raymond S. (1998), "Confirmation Bias: A Ubiquitous Phenomenon in Many Guises," Review of General Psychology, 2 (2), 175-220.

Piazza, Manuela, Veronique Izard, Philippe Pinel, Denis Le Bihan, and Stanislas Dehaene (2004), "Tuning Curves for Approximate Numerosity in the Human Intraparietal 
Sulcas," Neuron, 44 (10), 547-555.

Pinkley, R. L., T. L. Griffith, and G. B. Northcraft (1995), "Fixed Pie" a La Mode: Information Availability, Information Processing, and the Negotiation of Suboptimal Agreements, Organizational Behavior and Human Decision Processes, 62, 101-112.

Raghubir, Priya and Aradhna Krishna (1996), "As the Crow Flies: Bias in Consumers' Map-Based Distance Judgments," Journal of Consumer Research, 36 (10), 26-39. (1999), "Vital Dimensions in Volume Perception: Can the Eye Fool the Stomach?" Journal of Marketing Research, 36 (3), 313-326.

Redden, Joseph P. and Stephen J. Hoch (2009), "The Presence of Variety Reduces Perceived Quantity," Journal of Consumer Research, 23 (6), 406-417.

Schulz-Hardt, Stefan (1997), "Realitdtsflucht in Entscheidungsprozessen: Von Groupthink Zum Entscheidungsautismus [Loosing Reality in Decision Processes: From Groupthink to Autistic Decision Making]," Bern, Switzerland: Huber.

Schulz-Hardt, Stefan, Dieter Frey, C. Luthgens, and S. Moscovici (2000), "Biased Information Search in Group Decision Making," Journal of Personality and Social Psychology, 78, 655-669.

Snyder, M. and P. White (1981), "Testing Hypotheses About Other People: Strategies of Verification and Falsification," Personality and Social Psychology Bulletin, 7, 39-43. Tversky, Amos and Daniel Kahneman (1974), "Judgment Under Uncertainty: Heuristics and Biases," Science, 185 (9), 1124-1131. Vos, Piet G., Michiel P. van Oeffelen, Hein J. Tibosch, and Jüri Allik (1988), "Interactions Between Area and Numerosity," Psychological Research, 50, 148-154.

Yadav, Manjit S. (1994), "How Buyers Evaluate Product Bundles: A Model of Anchoring and Adjustment," Journal of Consumer Research, 21 (9), 342-353.

Yin, Dezhi, Sabyasachi Mitra, and Han Zhang (2016), "When Do Consumers Value Positive vs. Negative Reviews? An Empirical Investigation of Confirmation Bias in Online Word of Mouth," Information Systems Research, 27 (1), 131-144. 


\section{〈Appendix A〉 Pretest Measurement Questions}

\section{Common Belief or Expectation of Relationship between Variety and Quantity}

1. There are grocery stores, A and B. If store A offers more variety of products than store B, which store do you think contains a larger quantity of products that it stocks?

2. There are two restaurants, $A$ and $B$. If restaurant $A$ serves more variety of items on the menu than store $\mathrm{B}$, which restaurant do you think serves more meal?

3. There are two bookstores, A and B. If bookstore A offers more variety of categories of books than store $\mathrm{B}$, which bookstore do you think contains a larger quantity of books that it stocks?

4. There are two sportswear stores, $A$ and $B$. If sportswear store $A$ offers more variety of sports gears than store $B$, which store do you think contains a larger quantity of gears that it stocks?

5. There are two home supply stores, A and B. If sportswear store A offers more variety of sports gears than store $\mathrm{B}$, which store do you think contains a larger quantity of gears that it stocks?

\section{〈Appendix B〉 Scenario and Stimulus in Study 1 and Study 2}

\section{Written-Variety Condition}

On a weekday, you visit the stationery shop in your neighborhood to get some pens and notebooks. That day happens to be the $10^{\text {th }}$ anniversary of the store, and they are holding an anniversary event. Details about the event are below.

We are holding a draw to give away notebooks to 20 lucky winners who have spent over $\$ 10$, just for today. There are four notebooks of each color. We are giving away one of navy, red, green, grey, and purple notebooks for each winner.

\section{Written-No Variety Condition}

On a weekday, you visit the stationery shop in your neighborhood to get some pens and notebooks. That day happens to be the $10^{\text {th }}$ anniversary of the store, and they are holding an anniversary event. Details about the event are below.

We are holding a draw to give away notebooks to 20 lucky winners who have spent over $\$ 10$, just for today. We are giving away one navy notebook for each winner.

\section{Picture-Variety Condition and Picture-No Variety Condition}

On a weekday, you visit the stationery shop in your neighborhood to get some pens and notebooks. That day happens to be the $10^{\text {th }}$ anniversary of the store, and they are holding an anniversary event. Details about the event are below.

We are holding a draw to give away notebooks to 20 lucky winners who have spent over $\$ 10$, just for today. We are giving away one of the notebooks in the photo for each winner. 
Study 1 Picture-Variety Condition Stimuli

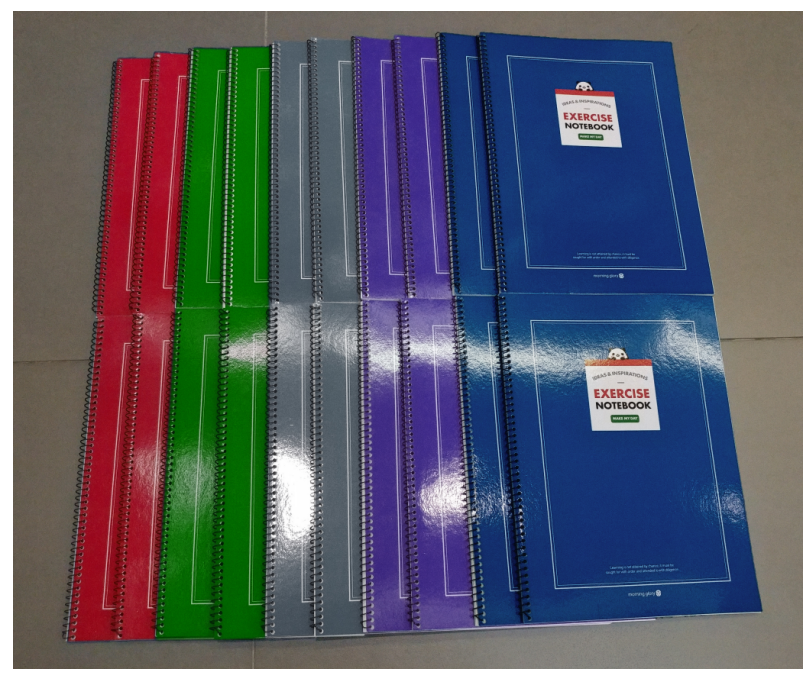

Study 1 Picture-No Variety Condition Stimuli

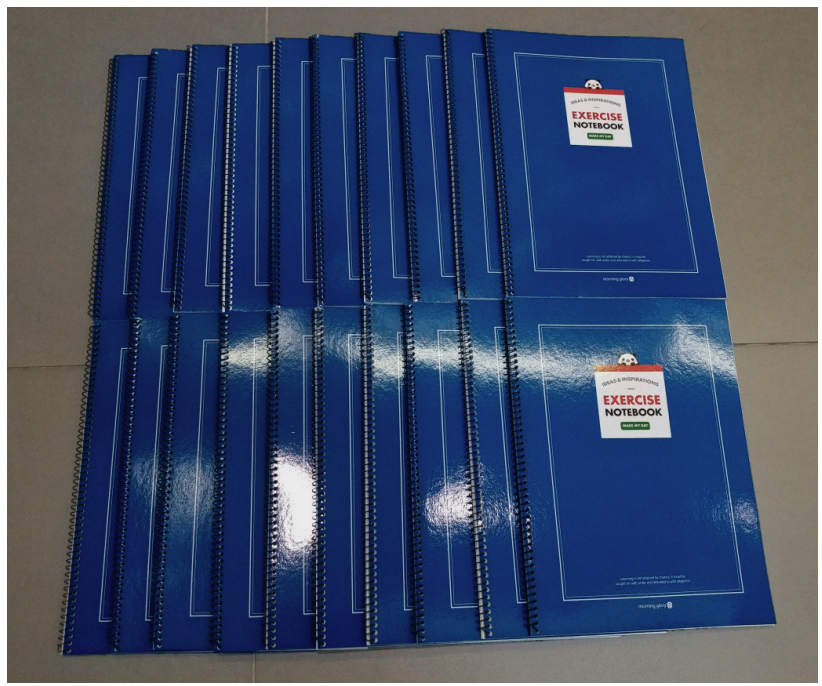


Study 2 Picture-Variety Condition Stimuli

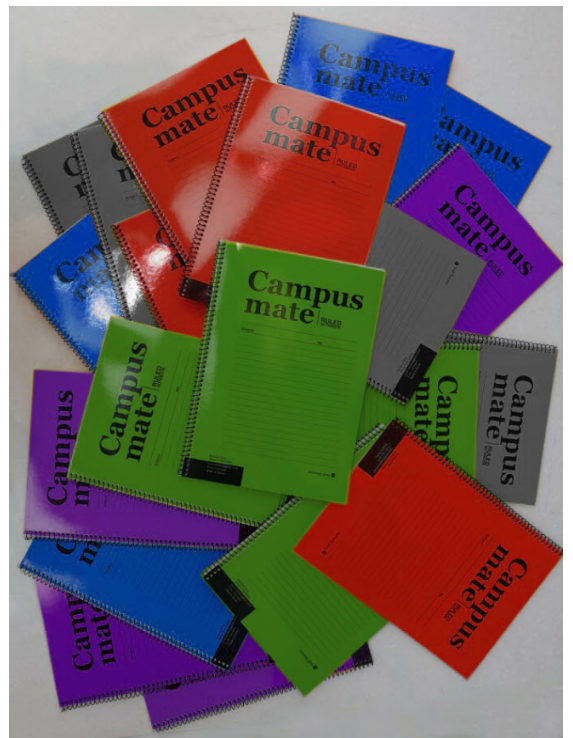

Study 2 Picture-No Variety Condition Stimuli

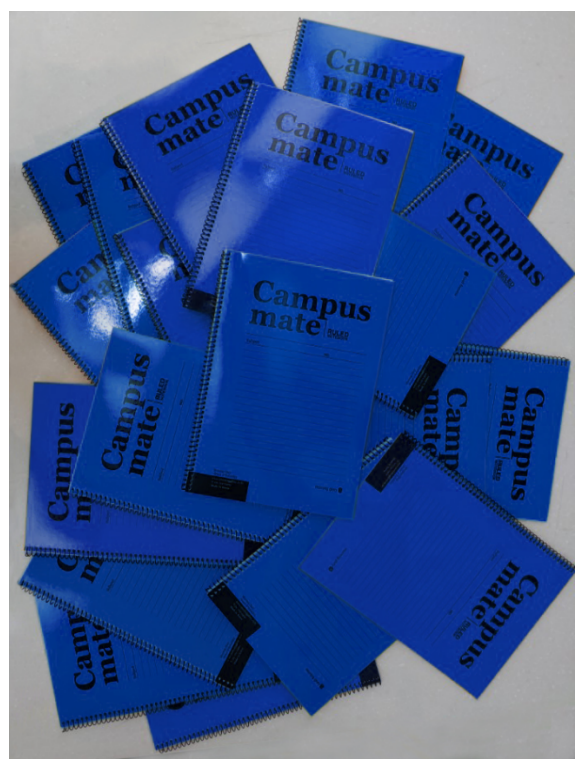

86 ASIA MARKETING JOURNAL Vol. 21 No. 01 April 2019 


\section{〈Appendix C〉 Variety Seeking Measurement Items}

Variety Seeking (Baumgartner and Steenkamp 1996; Donthu and Gilliland 1996; Jeong et al. 2016) 1. I like to try different things

2. I like a great deal of variety

3. I like new and different styles

4. I would rather stick with a brand I usually buy than try something I am not very sure of

5. When I go to a restaurant, I feel it is safer to order dishes I am familiar with

6. If I like a brand, I rarely switch from it just to try something different

7. I am very cautious in trying new or different products

8. Even though certain food products are available in a number of different flavors, I tend to buy the same flavor

9. I enjoy taking chances in buying unfamiliar brands to get some variety in my purchases 